\title{
Stress response pathways, toxicity pathways and adverse outcome pathways
}

\author{
Paul Jennings
}

Received: 11 October 2012/Accepted: 30 October 2012/Published online: 13 November 2012

(C) Springer-Verlag Berlin Heidelberg 2012

The aim of this editorial is to address ambiguities and misconceptions regarding the terms stress response pathways, toxicity pathways and adverse outcome pathways.

A large number of tightly regulated stress response pathways have evolved to allow cells to cope with and manage different types of cell stress. The purpose of these pathways is to maintain or reinstate homoeostasis in the face of perturbations of particular cellular processes. Here, I give a few well-characterised examples [for an in-depth review of these and other stress response pathways see (Jennings et al. 2012)]. The granddaddy of stress response pathways is the p53 pathway. It is considered a gatekeeper of DNA integrity, preventing cells with compromised DNA from proliferating further and thus can be considered a DNA damage stress response. Its activation induces the transcription of genes which, depending on the context lead to cell cycle arrest, senescence or apoptosis. Failure of the p53 pathway to engage correctly allows the propagation of cells with unstable DNA and is one of the major initiators of cancer development. The Nrf2 pathway is the main oxidative stress response and thus also of high importance to the field of toxicology. The activation of this pathway induces the transcription of genes involved in glutathione synthesis, glutathione recycling and detoxification. The lack of appropriate Nrf2 activation would result in a rapid depletion of glutathione leading to cell death at ordinarily tolerable concentrations of oxidant. This is illustrated in Nrf2 knockout mice, which are far more sensitive to

P. Jennings ( $\square)$

Division of Physiology, Department of Physiology and Medical Physics, Innsbruck Medical University, Fritz-Pregl Strasse 3/1, 6020 Innsbruck, Austria

e-mail: paul.jennings@i-med.ac.at; paul.other@gmail.com

URL: http://physiologie.i-med.ac.at/jennings acetaminophen than wild type mice (Enomoto et al. 2001). Harnessing the Nrf2 pathway also has therapeutic potential. Indeed, activation of Nrf2 via modification of its inhibitor KEAP-1 with bardoxolone methyl is in late clinical trials for the treatment of chronic renal disease progression (Crunkhorn 2012). The unfolded protein response (UPR) is activated by perturbations in the endoplasmic reticulum (ER) environment or due to protein overloading, collectively termed ER stress. The pathway temporarily shuts down protein translation and activates three transcriptional master regulators, ATF4, ATF6 and XBP1. These transcription factors have specific roles in the restoration of translation, increasing molecular chaperones and induction of both amino acid utilisation and aminoacyl tRNA synthetases. Several other stress response pathways have been identified, including the hypoxic stress response (regulated by hypoxia inducible factor) and the heavy metal stress response (regulated by metal responsive transcription factor). In the context of toxicology, it is important to remember that these stress response pathways are not the perturbation but the response to the perturbation.

A toxicity pathway was defined in the National Research Council's (NRC), Toxicity testing in the 21st century: a vision and a strategy, as "cellular response pathways that, when sufficiently perturbed in an intact animal, are expected to result in adverse health effects" (NRC 2007). This would by definition exclude stress response pathways as (1) their activation is a normal biological response to a specific stimulus not a perturbation and (2) they function to protect the cell or the tissue. However, I am certain the authors did not mean to exclude stress response pathways, especially since in a follow-up review the Nrf2 pathway was used to illustrate the toxicity pathway concept (Krewski et al. 2010). Adverse outcome pathways (AOPs) were first described in the field of ecotoxicology as "a 
conceptual construct that portrays existing knowledge concerning the linkage between a direct molecular initiating event and an adverse outcome at a biological level of organization relevant to risk assessment" (Ankley et al. 2010). Appling the concept to systemic toxicology, an adverse outcome in broad physiological terms is an event causing dysfunction of a tissue or organ where whole body homeostasis can no longer be maintained. Since the described stress response pathways aim to maintain homeostasis, they are not in themselves AOPs. Although, stress response pathways and/or toxicity pathways are built into the concept, as they provide valuable mechanistic information, which give clues to the initiating event. Thus, forgiving the poor definition of toxicity pathways, stress response pathways are encompassed by toxicity pathways, which are encompassed by AOPs.

Despite being able to rationalise these terms, I find the choice of wording unfortunate and unhelpful. Biologically speaking, a toxicity pathway or an adverse outcome pathway is meaningless as they would not be favoured by evolution. On the contrary, stress response pathways have evolved to overcome cellular perturbations and promote survival of the organism. I am often encountered by those who discount a specific stress response pathway as a protective adaptation and not a "real" toxicity pathway. It is also counter intuitive that a protective stress response can contribute to an "adverse outcome". How then are stress response pathways helpful in toxicity testing regimes and why should they be part of the toxicity pathway and adverse outcome pathway concepts? Identification of which pathways are activated by a particular compound tells us about the biological process which was perturbed. If the stress is increased (dose) or the duration of the stress is prolonged (exposure time), the pathway will be overwhelmed. Some pathways, such as p53 and UPR, have inbuilt mechanisms to initiate apoptosis. Apoptosis is, however, also not in itself an adverse outcome, as it is intended to protect the tissue from injury. If the pathway fails to redress the perturbation or initiate apoptosis, unregulated cell death will occur inevitably leading to tissue injury and disease (an adverse outcome).

Stress response pathways are of high relevance not only to the field of toxicology but to the entire biological spectrum. In cancer biology, for example, the inactivation and/or over activation of these pathways is fundamental to tumour development and survival. Genotoxic stress can act as a selection pressure for mutations in the p53 gene leading to p53 inactivation and tumour development (Stegh 2012). Permanent Nrf2 activation has also been shown to confer a survival advantage to cancer cells as they often face ischaemic environments (Sporn and Liby 2012). It is my view that the further identification and elucidation of stress response pathways are critical for the future of toxicology. The unravelling of their intricate interconnections will allow a much deeper mechanistic understanding of how cells react to cellular perturbations, whether they are brought about by internal or external stimuli. This represents a common goal in the fields of cellular physiology, molecular biology and molecular toxicology. In addition, a more in-depth knowledge of stress response pathways will not only provide us with the tools to develop superior models for safety assessment but will also open up new pharmaceutical targets for disease prevention and treatment.

\section{References}

Ankley GT, Bennett RS, Erickson RJ et al (2010) Adverse outcome pathways: a conceptual framework to support ecotoxicology research and risk assessment. Environ Toxicol Chem SETAC 29(3):730-741. doi:10.1002/etc.34

Crunkhorn S (2012) Deal watch: Abbott boosts investment in NRF2 activators for reducing oxidative stress. Nat Rev Drug Discov 11(2):96. doi:10.1038/nrd3655

Enomoto A, Itoh K, Nagayoshi E et al (2001) High sensitivity of Nrf2 knockout mice to acetaminophen hepatotoxicity associated with decreased expression of ARE-regulated drug metabolizing enzymes and antioxidant genes. Toxicol Sci Off J Soc Toxicol 59(1):169-177

Jennings P, Limonciel A, Felice L, Leonard MO (2012) An overview of transcriptional regulation in response to toxicological insult. Arch Toxicol. doi:10.1007/s00204-012-0919-y

Krewski D, Acosta D Jr, Andersen M et al (2010) Toxicity testing in the 21st century: a vision and a strategy. J Toxicol Environ Health Part B Crit Rev 13(2-4):51-138. doi:10.1080/10937404. 2010.483176

NRC (2007) Toxicity testing in the 21st century: a vision and a strategy. The National Academies Press, Washington. http:// www.nap.edu/openbook.php?record_id $=11970$

Sporn MB, Liby KT (2012) NRF2 and cancer: the good, the bad and the importance of context. Nat Rev Cancer 12(8):564-571. doi: $10.1038 / \mathrm{nrc} 3278$

Stegh AH (2012) Targeting the p53 signaling pathway in cancer therapy-the promises, challenges and perils. Expert Opin Ther Targets 16(1):67-83. doi:10.1517/14728222.2011.643299 